\title{
Efficacy and safety of nivolumab plus apatinib in advanced liver carcinosarcoma: a case report
}

\author{
Lingdi Zhao', Yonghao Yang1 \& Quanli Gao*,1 \\ ${ }^{1}$ Department of Immunotherapy, Affiliated Cancer Hospital of Zhengzhou University \& Henan Cancer Hospital, Zhengzhou City, \\ Henan Province, China \\ *Author for correspondence: gaoquanli2015@126.com
}

\begin{abstract}
Liver carcinosarcoma is a rare malignancy usually seen in adults, majority of whom have a history of chronic hepatitis. Radical resection in early stages leads to better prognosis; however, the treatment regimen for patients at advanced stage is usually based on previously published case reports. At present, there is no report on programmed death-1 blockade and liver carcinosarcoma. Here, we report the case of a patient with advanced liver carcinosarcoma treated with nivolumab plus apatinib, which resulted in partial remission. However, grade 3 elevation of aminotransferase occurred during treatment, suggesting that the combination therapy should be recommended only after risk assessment. Nonetheless, programmed death-1 blockade plus apatinib might be a promising therapeutic approach for patients with advanced liver carcinosarcoma.
\end{abstract}

First draft submitted: 22 December 2018; Accepted for publication: 2 April 2019; Published online: 15 May 2019

Keywords: apatinib • carcinosarcoma • combination • immunotherapy • nivolumab

Carcinosarcoma is often referred to a malignant mixed tumor with high invasiveness. Liver carcinosarcoma is a rare malignancy that was first defined by Craig et al. as hepatic tumors with both hepatocellular carcinoma and a nonspindle cell sarcoma with specialized differentiation, such as malignant skeletal muscle, bone or cartilage [1]. Owing to the nonspecificity of image examination and the limitation of biopsy, it is very difficult to get an accurate diagnosis before surgery. The sarcoma element in liver carcinosarcoma possesses high invasiveness, and the prognosis of patients with liver carcinosarcoma is worse than those with primary hepatocellular carcinoma and cholangiocarinoma [2]. Radical surgery is the only possible method for cure in patients with early stage [3]; however, recurrence occurs soon after the previous surgery. Most patients could not achieve radical resection because of extrahepatic metastasis, vascular invasion and involvement of surrounding organs. Even among those patients who can receive surgery resection, most die within 1 year due to the recurrence. The median survival time of patients with metastatic diseases is only about 6 months [4]. Conducting clinical studies in liver carcinosarcoma is very difficult due to the low incidence of this malignancy, and the treatment experiences of such patients from case reports or case series are very important in choosing regimen for patients with advanced diseases. Here, we report the treatment of a patient with liver carcinosarcoma with the simultaneous application of nivolumab and apatinib and the overall survival time for this patient was 12 months.

\section{Case presentation}

A 57-year-old man had a history of hepatitis B for more than 10 years. In December 2016, there was a tumor in his liver identified by medical examination. Radical resection was conducted in December 2016. There were no ascites, stellate nodes and portal vein tumor thrombi. The resected tumor was macroscopically a simple nodular type, $10 \times 8 \times 4 \mathrm{~cm}^{3}$ in maximum cross-section, located at segments IV and V of liver, showed involvement of liver capsule, no invasion of nerve and vessels and no tumor cells at margins of excision. Immunohistochemistry showed that the tumor cells were positive for CK, Vim, CK8/18 and CD34; weakly positive for programmed death ligand 1 (PD-L1); and negative for SMA, Des, CK7, CD68 and CD163. The postoperative pathological 

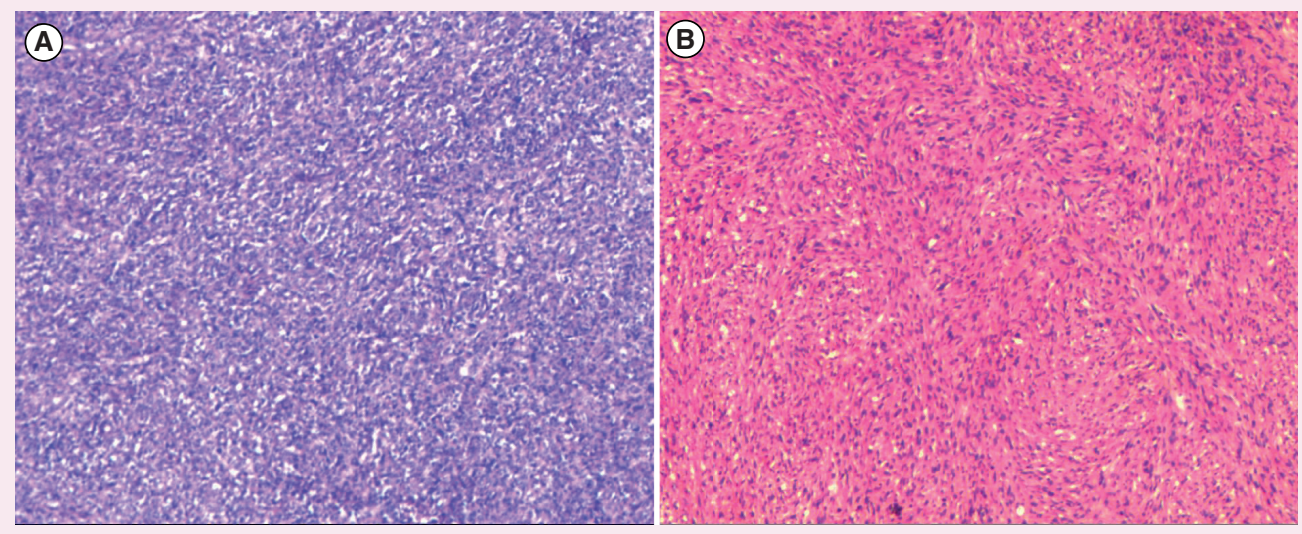

Figure 1. Histopathological hematoxylin-eosin staining images (both the images were in low magnification). (A) The histopathological image after surgery, cancer components and sarcomatoid components could be seen on the image and (B) is the histopathological image at the beginning of relapse, sarcomatoid components increased in the image.

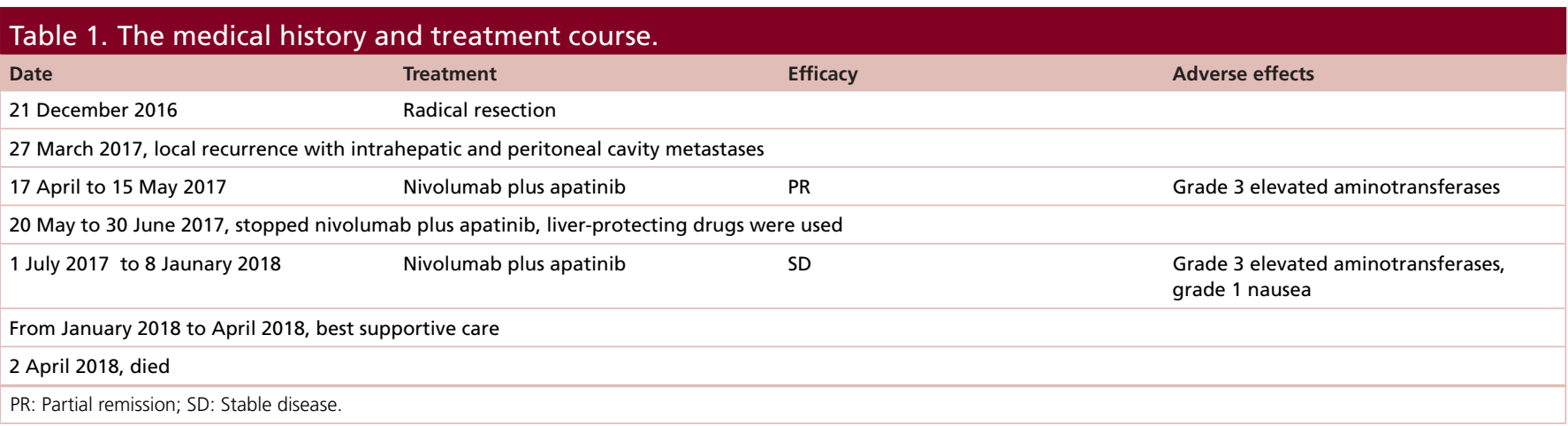

diagnosis was liver carcinosarcoma. Figure 1A was the hematoxylin-eosin (HE) staining result. The wound heals well and he was discharged from hospital 16 days after surgery. He felt abdominal distension and a computed tomography was performed on 27 March 2017. The results showed local recurrence with intrahepatic and peritoneal cavity metastases. Liver biopsy was performed and the pathology showed spindle cell tumor with cancerous differentiation (Figure 1B), together with the past medical history, consistent with liver carcinosarcoma, suggesting immunohistochemical examination for confirmation. The patient refused immunohistochemical examination, and the biomarkers were not rechecked. The patient refused chemotherapy and minimally invasive surgery. His symptom of abdominal distension became progressive. A computed tomography after half a month showed the tumors had become enlarged (Figure 2A-C). With the consent of the patient, he was administered an off-label nivolumab plus apatinib. The nivolumab was given by intravenous injection at the dose of $3 \mathrm{mg} / \mathrm{kg}$ biweekly while apatinib was given by oral administration at the dose of $500 \mathrm{mg}$ daily. After two doses of nivolumab, the tumors shrank slightly, and the density of the tumors reduced (Figure 2D-F). After four doses of nivolumab, grade 3 elevation of alanine aminotransferase and aspartate aminotransferase occurred. Since there was no evidence of HBV replication, the elevation of the aminotransferases was ascribed to the drugs, either nivolumab or apatinib, or both, after which the drugs were discontinued. At the same time, the patient received liver-protecting drugs with magnesium isoglycyrrhizinate injection and transmetil. After 3 weeks the aminotransferases decreased to normal levels and the nivolumab plus apatinib therapy was resumed; at the same time, diammonium was used for prevention of liver damage. 1 month later, the tumors shrank and partial remission was considered using RECIST 1.1 standard (Figure 2G-I). 3.5 months later, the therapy was discontinued again because of the grade 3 elevation of aminotransferases, during which the tumors were stable. The disease had progressed when examined on January 2018 and the treatment regimen was switched to best supportive care. The patient died on 2 April 2018. The medical history and therapeutic course were listed at Table 1. As this patient died, the patient's son signed an 

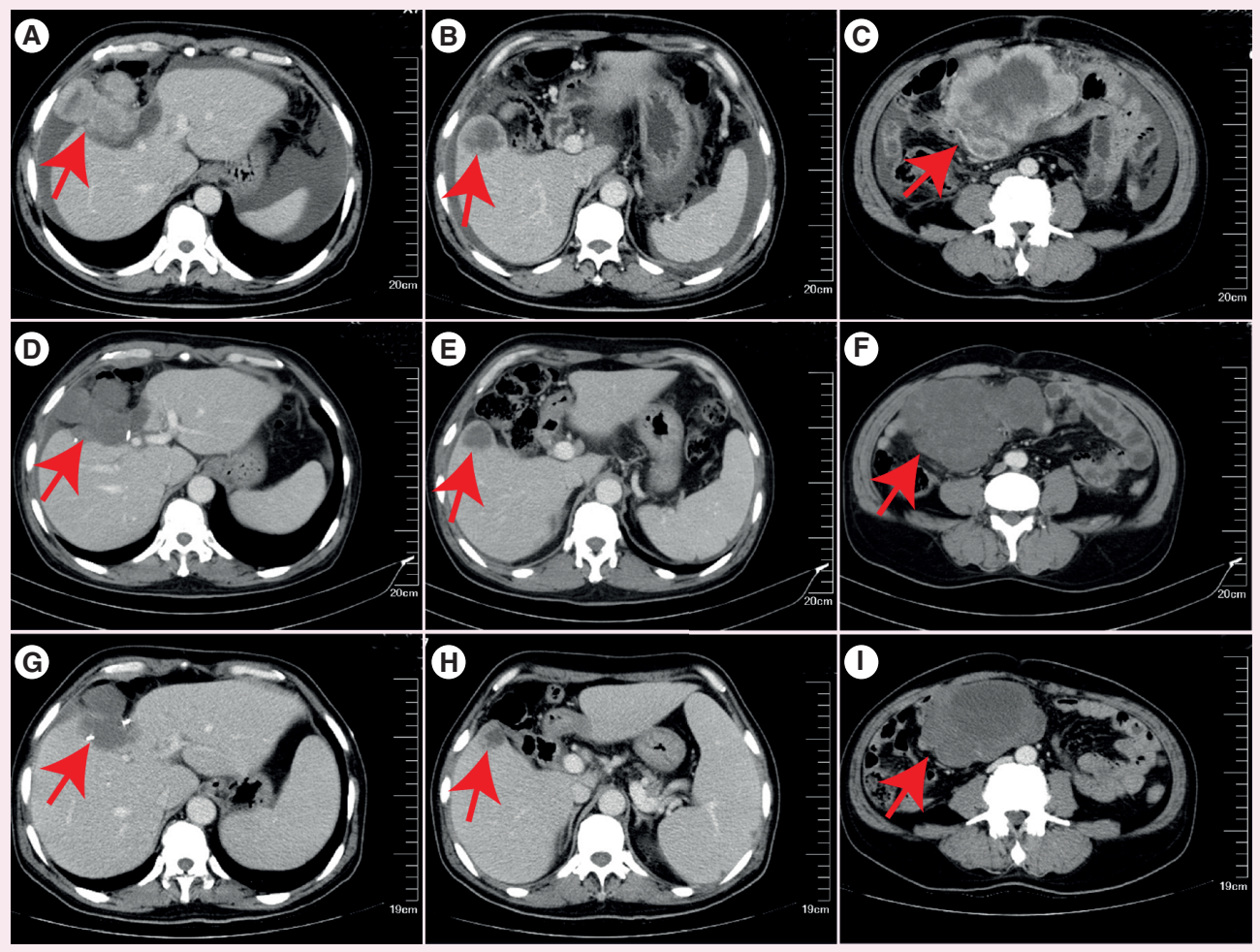

Figure 2. Enhanced computed tomography of the tumors (plain scans and multiphase enhanced scans including arterial phase, portal phase and equilibrium phase were performed each time. All the scans chosen in the figure were arterial phase imaging). (A-C) Show the lesions in the local relapse, intrahepatic and peritoneal cavity metastasis. (D-F) Show the shrunk lesions after 20 days of therapy with nivolumab and apatinib. (G-I) Show the further shrinkage of the lesions after about 2 months of combined therapy.

approval for the publication of the cases.

\section{Discussion}

The WHO defines liver carcinosarcoma as a malignant tumor containing an intimate mixture of carcinomatous elements (either hepatocellular carcinoma or cholangiocarcinoma) and sarcomatous elements [5]. This definition was widely accepted, as it does not emphasize the specialized differentiation of the sarcomatous components. As the low incidence of liver carcinosarcoma, the pathogenesis is controversial. One view is that liver carcinosarcoma is of monoclonal origin. It was reported that most cases originated from normal hepatocyte without cirrhosis, supporting the hypothesis that liver carcinosarcoma begins from a pluripotent precursor cell or stem cell, then differentiates into carcinomatous element and sarcomatous element [6]. Others argue that primary hepatocellular carcinoma transforms into mesenchymal component; the presence of transitional or transformational zones between carcinoma and sarcoma in some cases supports this view [7-9]. According to the study by Yamamoto et al., which analyzed the reports published before 2003, more than half of the liver carcinosarcoma patients had a medical history of chronic viral hepatitis or cirrhosis [2,4], which supports that the opinion of the latter. Although there have been many studies of predictive and prognostic biomarkers in liver cancer (including primary liver cancer and metastatic liver cancer) [10], there are still no prognostic biomarkers studied in liver carcinosarcoma.

Radical resection is recommended to be the standard treatment for patients whose disease is at an early stage [11]. Chemotherapy or targeted therapy such as etoposide, cisplatin, carboplatin, mitomycin and sorafenib could be applied to patients whose diseases cannot be treated by radical resection [4]. Although previous studies report the prolonged progression-free survival by postoperative concurrent chemoradiotherapy, the poor tolerance for such therapy makes it unsuitable for most patients [12]. Hepatitis virus infection can cause injury to the liver, resulting in the reduced liver reserve function. Most drugs are metabolized by the liver, and some antitumor drugs lead to reactivation of the hepatitis virus, which could aggravate liver injury. There have been many reports in the 
literature that chemotherapy and rituximab cause the outbreaks of hepatitis B. Immunotherapeutic drugs and many small molecule targeted therapeutic drugs are harmful to the liver and incite abnormal liver function; under the background of viral hepatitis the hepatic dysfunction would be more severe. Immune checkpoint inhibitors reactivate cytotoxic $\mathrm{T}$ cells, these cells may have a beneficial role in the treatment of chronic hepatitis; however, hepatocellular injury caused by an exaggerated immune response is an issue of concern. Exploring new approaches for treating this rare disease is therefore of great significance, especially for the patients whose disease is at late stage.

Nivolumab is an anti-programmed-death-1 (PD-1) immunoglobin G4 monoclonal antibody, which has been added to the treatments of many different types of cancer [13]. There are studies reporting the efficacy of PD-1 blockade and hepatocellular carcinoma [14,15]. Nivolumab binds to PD-1 and blocks the interaction with PD-L1 and PD-L2, preventing T-cell inhibition and restoring the antitumor responses of $T$ cells $[13,16]$. There are also reports of efficacy of PD-1 blockade in pulmonary carcinosarcoma, ovarian carcinosarcoma and uterine carcinosarcoma [17-19]. However, to date, the studies on the efficacy of PD-1 blockade in liver carcinosarcoma are rare.

Apatinib is an oral small molecule VEGFR2 inhibitor and shows antitumor activity in different kinds of tumors including soft tissue sarcoma [20]. Anti-angiogenesis could regulate the tumor microenvironment by myeloidderived suppressor cell, regulatory $\mathrm{T}$ lymphocyte, tumor-associated macrophage and cytotoxic T lymphocyte [21]. Successful reversal of immune evasion can be achieved by checkpoint inhibitors, particularly in cancers with high-immunogenic potential. Theoretically, anti-angiogenesis may play a synergistic role with anti-PD-1 therapy. Indeed, synergy of anti-PD-1 therapy and anti-angiogenesis therapy have been demonstrated in lung cancer and renal cell carcinoma [22,23]. Moreover, PD-1 blockade plus apatinib has manifested promising antitumor activity in hepatocellular carcinoma, gastric or esophagogastric junction cancer [24].

So far, there is little data on anti-PD-1 therapy plus anti-angiogenesis therapy in liver carcinosarcoma. Although this patient received radical surgery and R0 resection, the tumor involved the liver capsule and the high-grade biological character of the tumor resulted in relapse and metastasis within 4 months after the surgery, by which time the tumors had enlarged quickly. The tumor cells were weakly positive for PD-L1, hinting that the patient might benefit from anti-PD-1 therapy. Anti-PD-1 therapy displays antitumor effect by regulating the immune system, which needs relatively long time. Additionally, since nivolumab alone could not shrink the tumors in a short time, a combination of nivolumab and apatinib was advised for the patient. After 20 days of combined therapy, the tumors became smaller as shown in Figure 2D-F. As seen in Figure 2, mild splenomegaly appeared 1 month later and kept at slightly enlarged level. We regarded it as congestive splenomegaly caused by compression of abdominal mass. During the treatment, the diseases shrank gradually, and partial remission was achieved. At the same time, the symptom of abdominal distension improved. It should be noted that the therapy was discontinued twice due to grade 3 elevation of aminotransferases. History of viral hepatitis over 10 years and mild cirrhosis before treatment indicated poor liver compensatory function, and close monitoring liver function during treatment was very important.

\section{Conclusion}

Liver carcinosarcoma is a rare malignancy with high aggression. Currently the treatment options for advanced liver carcinosarcoma are usually derived from the experiences of accumulation of case reports. With the advent of the era of immunotherapy, immune checkpoint inhibitors may provide benefit for such patients, especially under the guidance of biomarkers such as PD-L1 expression, tumor mutation burden, microsatellite stability, etc. However, it usually takes a long time for the immune checkpoint inhibitor to be effective, immune checkpoint inhibitor alone is usually not enough for the patients whose disease grows fast, which the rapid tumor shrinkage is important for the patients to relieve symptoms. For patients whose tumors grow fast, immune checkpoint inhibitors combined with other treatment modes, such as anti-angiogenesis therapy, chemotherapy, radiotherapy, is needed. The clinical efficacy outlined in this case report suggests that nivolumab plus apatinib for metastatic liver carcinosarcoma might prolong survival time. However, the risk of adverse events due to this combined regimen especially in patients with the history of chronic hepatitis should be carefully considered. The accumulation of case reports will be beneficial to determine the appropriate treatment for patients with liver carcinosarcoma.

\section{Future perspective}

The pathogenesis of malignancies is closely related to the imbalance of immune system, immune checkpoint inhibitors represented by PD-1 inhibitors have shown a broad spectrum antitumor effect in clinic. Currently immunotherapy is becoming more and more important in the multidisciplinary treatment for cancer. However, 
the overall response rate of immune checkpoint inhibitors is usually low, immunotherapy under the guidance of biomarkers or combined immunotherapy has become one of the research hotspots in cancer therapy at present. In the future, the cancer patients will be classified according to the gene mutation status, fusion gene, tumor mutation load, microsatellite stability, expression of mismatch repair protein and PD-L1, etc. and will be treated accordingly. The classification of malignancies would not be limited to the location and tissue origin of the tumors. The classification of malignancies based on genetic changes or protein expressions in tumors may provide the possibility of conducting clinical studies in rare tumors such as liver carcinosarcoma, making it possible to provide treatment recommendations for these kinds of patients.

\section{Executive summary}

- Liver carcinosarcoma is an uncommon maligant tumor originating from the liver, which possesses high invasiveness and poor prognosis. There are no effective standard regimens for patients with advanced liver carcinosarcoma at present. The current clinical regimens of patients with such kind of diseases usually come from the experience of the doctors and the experiences from case reports. It is very important to explore the treatment regimens for patients with advanced liver carcinosarcoma.

- Nivolumab is a monoclonal antibody targeting programmed death-1 molecule and has been shown good clinical efficacy in a variety of solid tumors. However, the overall efficacy of nivolumab is low, nivolumab needs to be combined with other regimens to improve its efficacy. Apatinib is a small molecule that possesses antiangiogenic effect. Apatinib has been shown immunomodulatory effects in preclinical studies. In patients with advanced liver cancer, apatinib and SHR-1210 (another anti-programmed-death-1 antibody) has shown good clinical efficacy.

- A 57-year-old man was diagnosed with advanced liver carcinosarcoma and was treated with nivolumab plus apatinib. After 1 month of therapy tumor shrinkage occurred and the clinical symptoms were alleviated. During treatment partial remission was got. However, treatment was discontinued twice during therapy as grade 3 elevated transaminase.

\section{Financial \& competing interests disclosures}

The authors have no relevant affiliations or financial involvement with any organization or entity with a financial interest in or financial conflict with the subject matter or materials discussed in the manuscript. This includes employment, consultancies, honoraria, stock ownership or options, expert testimony, grants or patents received or pending, or royalties.

No writing assistance was utilized in the production of this manuscript.

\section{Ethical conduct of research}

The authors state that they have obtained appropriate institutional review board approval or have followed the principles outlined in the Declaration of Helsinki for all human or animal experimental investigations. In addition, for investigations involving human subjects, informed consent has been obtained from the participants involved.

\section{Informed consent disclosure}

The authors state that they have obtained verbal and written informed consent from the patient/patients for the inclusion of their medical and treatment history within this case report.

\section{References}

1. Craig JR, Peters RL, Edomondson HA. Tumors of the Liver and Intrahepatic Bile Ducts. Armed Forces Institute of Pathology. DC, USA (1989).

2. Li J, Liang P, Zhang D et al. Primary carcinosarcoma of the liver: imaging features and clinical findings in six cases and a review of the literature. Cancer Imaging 18(1), 7 (2018).

3. Goto H, Tanaka A, Kondo F et al. Carcinosarcoma of the liver. Intern Med. 49(23), 2577-2582 (2010).

4. Yamamoto T, Kurashima Y, Ohata K et al. Carcinosarcoma of the liver: report of a case. Surg. Today 44(6), 1161-1170 (2014).

5. Ishak KG, Anthony PP, Niederau C et al. WHO international histological classification of tumours, pathology \& genetics of the tumours of the digestive system. In: Mesenchymal Tumours of the Liver. IARC Press. Lyon, France198 (2000).

6. Fayyazi A, Nolte W, Oestmann JW et al. Carcinosarcoma of the liver. Histopathology 32(4), 385-387 (1998).

7. Nomura K, Aizawa S, Ushigome S. Carcinosarcoma of the liver. Arch. Pathol. Lab. Med. 124(6), 888-890 (2000).

8. She R, Szakacs J. Carcinosarcoma of the liver: a case report and review of the literature. Arch. Pathol. Lab. Med. 129(6), 790-793 (2005). 
9. Gu YJ, Zhu YY, Lu XY, Zhao Q, Cong WM. Hepatic carcinosarcoma: evidence of polyclonal origin based on microsatellite analysis. Pathol. Res. Pract. 211(12), 905-910 (2015).

10. Golubnitschaja O, Polivka J Jr, Yeghiazaryan K, Berliner L. Liquid biopsy and multiparametric analysis in management of liver malignancies: new concepts of the patient stratification and prognostic approach. EPMA J. 9(3), 271-285 (2018).

11. Lao XM, Chen DY, Zhang YQ et al. Primary carcinosarcoma of the liver: clinicopathologic features of 5 cases and a review of the literature. Am. J. Surg. Pathol. 31(6), 817-826 (2007).

12. Kurita D, Mokuno Y, Matsubara H et al. Primary hepatic carcinosarcoma with multimodal treatment. Nagoya J. Med. Sci. 80(3), 423-429 (2018).

13. Li Y, Li F, Jiang F et al. A mini-review for cancer immunotherapy: molecular understanding of PD-1/PD-L1 pathway \& translational blockade of immune checkpoints. Int. J. Mol. Sci. 17(7), pii: E1151 (2016).

14. El-Khoueiry AB, Sangro B, Yau T et al. Nivolumab in patients with advanced hepatocellular carcinoma (CheckMate 040): an open-label, non-comparative, Phase 1/2 dose escalation and expansion trial. Lancet 389(10088), 2492-2502 (2017).

15. Kudo M. Systemic therapy for hepatocellular carcinoma: latest advances. Cancers (Basel) 10(11), pii: E412 (2018).

16. Guo L, Zhang H, Chen B. Nivolumab as programmed death-1 (PD-1) inhibitor for targeted immunotherapy in tumor. J. Cancer 8(3), 410-416 (2017).

17. Bhangoo MS, Boasberg P, Mehta P et al. Tumor mutational burden guides therapy in a treatment refractory POLE-mutant uterine carcinosarcoma. Oncologist 23(5), 518-523 (2018).

18. Zibetti Dal Molin G, Abrahao CM, Coleman RL, Maluf FC. Response to pembrolizumab in a heavily treated patient with metastatic ovarian carcinosarcoma. Gynecol. Oncol. Res. Pract. 5, 6 (2018).

19. Zhang Z, Chen Y, Ma M et al. Significant benefit of nivolumab treating PD-L1 positive metastatic pulmonary carcinosarcoma: a case report and literature review. Oncotarget 8(56), 96453-96459 (2017).

20. Xie L, Guo W, Wang Y, Yan T, Ji T, Xu J. Apatinib for advanced sarcoma: results from multiple institutions' off-label use in China. BMC Cancer 18(1), 396 (2018).

21. Li YL, Zhao H, Ren XB. Relationship of VEGF/VEGFR with immune and cancer cells: staggering or forward? Cancer Biol. Med. 13(2), 206-214 (2016).

22. Atkins MB, Plimack ER, Puzanov I et al. Axitinib in combination with pembrolizumab in patients with advanced renal cell cancer: a non-randomised, open-label, dose-finding, and dose-expansion Phase 1b trial. Lancet Oncol. 19(3), 405-415 (2018).

23. Socinski MA, Jotte RM, Cappuzzo F et al. Atezolizumab for first-line treatment of metastatic nonsquamous NSCLC. N. Engl. J. Med. 378(24), 2288-2301 (2018).

24. Xu JM, Zhang Y, Jia R et al. Anti-PD-1 antibody SHR-1210 combined with apatinib for advanced hepatocellular carcinoma, gastric or esophagogastric junction cancer: an open-label, dose escalation and expansion study. Clin. Cancer Res. 25(2), 515-523 (2019). 